\title{
Conceptual Design of the Beamline for the DTT Neutral Beam Injector following a Double Beam Source Design Approach ${ }^{*)}$
}

\author{
Piero AGOSTINETTI ${ }^{1)}$, Eugenio BENEDETTI ${ }^{2)}$, Tommaso BOLZONELLA ${ }^{1)}$, \\ Massimiliano BONESSO ${ }^{3,4)}$, Irene CASIRAGHI ${ }^{5,6)}$, Razvan DIMA ${ }^{3)}$, Giacomo FAVERO $^{3)}$, \\ Alberto FERRO ${ }^{1)}$, Marco GOBBIN ${ }^{1)}$, Gustavo GRANUCCI ${ }^{5)}$, Chundong HU $^{7)}$, Paola MANTICA ${ }^{5)}$, \\ Francesco LUCCHINI ${ }^{1,8)}$, Adriano PEPATO ${ }^{3)}$, Nicola PILAN ${ }^{1)}$, Fabrizio RAFFAELLI ${ }^{2)}$, \\ Pietro REBESAN ${ }^{3,9)}$, Afra ROMANO ${ }^{10,11)}$, Gianluca SPIZZO ${ }^{1)}$, Fabio VERONESE ${ }^{1,8}$, \\ Pietro VINCENZI ${ }^{1)}$, Yahong $\mathrm{XIE}^{7)}$ and Yuanlai XIE ${ }^{7)}$ \\ ${ }^{1)}$ Consorzio RFX (CNR, ENEA, INFN, Università di Padova, Acciaierie Venete SpA), C.so Stati Uniti 4, 35127 Padova, Italy \\ ${ }^{2)}$ INFN-Section of Pisa - Polo Fibonacci, Largo B. Pontecorvo 3, 56127 Pisa, Italy \\ ${ }^{3)}$ INFN-Section of Padova, Via Marzolo 8, 35131 Padova, Italy \\ ${ }^{4)}$ Dept. of Industrial Engineering, University of Padova, Via Marzolo 9, 35131 Padova, Italy \\ ${ }^{5)}$ Institute for Plasma Science and Tecnology, Via R. Cozzi 53, 20125 Milano, Italy \\ ${ }^{6)}$ Dept. of Physics G. Occhialini, Milano-Bicocca University, Piazza della Scienza 3, 20126 Milano, Italy \\ ${ }^{7)}$ Institute of Plasma physics CAS, Hefei, Anhui 230031, China \\ ${ }^{8)}$ Dept. of Electrical Engineering, University of Padova, Via Gradenigo, 6/A, Italy \\ ${ }^{9)}$ Dept. of Mechanical Engineering, Politecnico di Milano, via La Masa 1, 20156 Milano, Italy \\ ${ }^{10)}$ DTT S.c.a.r.l., Consorzio per l'attuazione del Progetto Divertor Tokamak Test, v. E. Fermi, 4500044 Frascati, Italy \\ ${ }^{11)}$ ENEA, Fusion and Technologies for Nuclear Safety Dept, C.R. Frascati, C.P. 6500044 Frascati, Italy
}

(Received 19 November 2020 / Accepted 13 April 2021)

\begin{abstract}
The main purpose of the Divertor Tokamak Test facility (DTT) [1], whose construction is starting in Frascati, Italy, is to study solutions to mitigate the issue of power exhaust in conditions relevant for ITER and DEMO. DTT will be equipped with a significant amount of auxiliary heating power ( $45 \mathrm{MW}$ ) to reach $P_{\mathrm{SEP}} / R=15 \mathrm{MW} \mathrm{m}^{-1}$ required to be DEMO-relevant [2]. DDT is characterized by high flexibility for the assembling and testing of divertor components and for the different magnetic configurations to address the integrated physics and technology problems. The current conceptual design of the beamline for the DTT Neutral Beam Heating system is here presented, with a particular focus on the effect on the DTT plasma and on the technical solutions adopted to maximize the RAMI indexes (Reliability, Availability, Maintainability and Inspectability) and minimize complexity and costs. Various design options were considered, and a comprehensive set of simulations was carried out using several physics and engineering codes to drive the choice of the most suitable design options and optimize them, aiming at finding a good compromise among different requirements. This paper describes the design of the main components of the DTT NBI beamline, explaining the motivations for the main design choices.
\end{abstract}

(c) 2021 The Japan Society of Plasma Science and Nuclear Fusion Research

Keywords: DTT neutral, beam, injector, conceptual, design

DOI: $10.1585 /$ pfr.16.2405080

\section{Introduction}

The Divertor Tokamak Test facility (DTT) aims at exploring alternative power exhaust solutions for the machines that will follow ITER [3,4], in particular the demonstrative power plant DEMO that is currently in the preconceptual design phase [5]. Namely, the principal objective of DTT is to mitigate the risk of a difficult extrapolation to a fusion reactor of the conventional divertor based on detached conditions, under test for ITER. The task, including several issues, has the main target to study

author'se-mail: piero.agostinetti@igi.cnr.it

*) This article is based on the presentation at the 29th International Toki Conference on Plasma and Fusion Research (ITC29). the completely integrated (physics-technology and bulkedge) power exhaust problems and to demonstrate how the possible implemented solutions (e.g., advanced divertor configurations or liquid metals) can be integrated in the DEMO device and other fusion reactors. DTT transport analysis and energetic particles studies have shown that a neutral beam with $510 \mathrm{keV}$ and $10 \mathrm{MW}$ injected to the plasma, compared to the previous solution with two beams of $400 \mathrm{keV}, 7.5 \mathrm{MW}$ each [6, 7], allows for significant improvements regarding plasma energy transport and fast particles dynamics, including the possibility of investigating fast ion physics in super-alfvenic scenarios. 


\section{Effects of the NBI on the Plasma}

METIS fast tokamak simulator [8] has been used to simulate DTT reference single null plasma scenario, including the single, high energy injector option with an injection angle of $35^{\circ}$ (equivalent to a tangency radius of $1.91 \mathrm{~m}$ ). Plasma heating for $500 \mathrm{keV}$ option is shown in Fig. 1 (a), including the ratio to electron and ions. METIS simulations supported the current choice of NBI parameters, in terms of central heating requirement, and avoidance of large shine-through losses for the reference, highdensity plasma scenario. In Fig. 1 (b), we compared the current NBI setting with lower energy and power options. Due to design flexibility, reduced energy and power NBI is possible for DTT, being this relevant for lower density plasma scenarios. The tangential injection allows also current drive, as shown in Fig. 1 (c).

Fast ion first-orbit losses and orbit losses due to magnetic field ripple have been estimated with ORBIT code [9]. Initial 3D ion positions have been calculated via the code TRANSP [10] considering the reference single null scenario, and given as input to ORBIT. By plotting the fast particle loss times (in toroidal transits $T$ ) as a function of pitch $\lambda=v_{\|} / v$, one can easily recognize prompt losses (loss times $<10 T$ ) and resonant losses (loss times $>10 T$ ): this is shown in Fig. 1 (d). Resonant losses are present only when ripple is switched on, and have a characteristic, distinct value of pitch $\lambda \sim 0.65$ : this behaviour is consistent
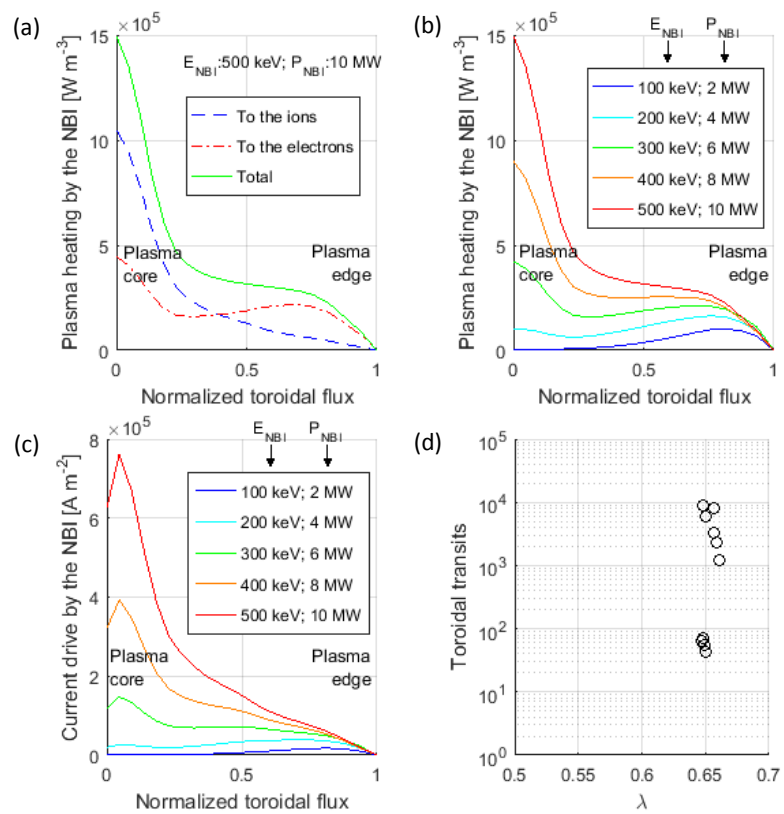

Fig. 1 Main physics parameters leading the design, evaluated using the METIS and ORBIT codes: (a) heating to the ions and electrons of the plasma; (b) plasma heating by the NBI as a function of beam energy and power; (c) current drive by the NBI as a function of beam energy and power; (d) toroidal turns travelled by the particles before being lost, as a function of the pitch parameter $\lambda$ (prompt losses are zero while resonant losses are $0.11 \%$ ). with the theory of ripple-precession resonance [11]. All losses are anyway found to be very small: prompt losses are zero, due to the fact that the beam is well aligned at $\theta=0$, while ripple (resonant) losses are only $0.11 \%$. With respect to previous estimates [7], ripple losses are similar $(0.15 \%$ in the old runs), while prompt losses pass from $3.85 \%$ to zero. This might be due to the fact that initial positions were calculated with METIS in 1D, and the angular positions were extrapolated from similar runs on ASDEXUpgrade [12]. The use of a full, 3D set of initial positions with TRANSP allows for a much more reliable calculation of prompt losses, showing at the same time that rippleresonant losses are instead dependent mostly on the radial position of injected particles.

\section{Main Design Features}

An overall view of the current conceptual design of the injector is given in Fig. 2 (a). As for the NBIs of JT60 [13] and LHD [14], a design with an air-insulated beam source is adopted. This solution was selected because it

(a)

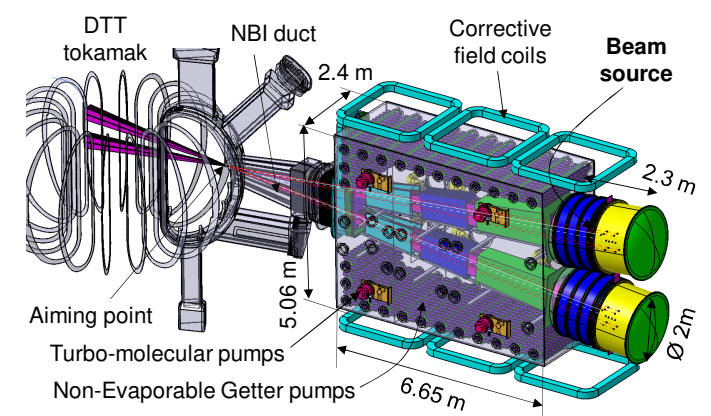

(b)

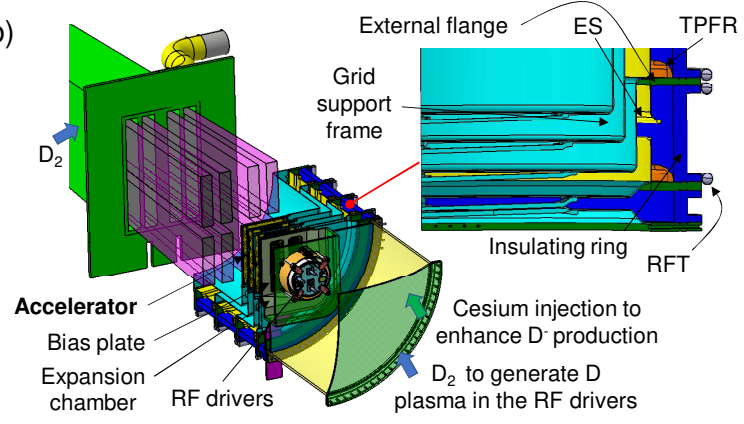

(c)

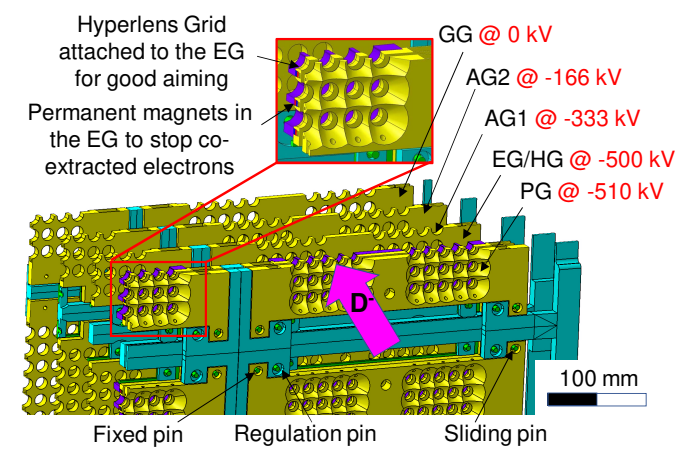

Fig. 2 Conceptual design of the beamline for DTT NBI: (a) overall view; (b) Beam source section view; (c) accelerator section view. 
maximizes the Reliability and Availability indexes, by improving the beam source accessibility and by avoiding the need of a single large bushing to connect the Transmission Line to the vacuum vessel. A drawback of this solution is the required clearance between the ion source and the surrounding building structures (at least $2.5 \mathrm{~m}$ with $510 \mathrm{kV}$, to avoid electrical discharges). The DTT design differs from the Japanese scheme in the choice of the ion source: in fact, as shown in Fig. 2 (b) it is proposed to use the same Radio Frequency source concept adopted for ITER, developed by IPP Garching [15]. The Beam Line Components (BLC), i.e. the Neutralizer, the Residual Ion Dump (RID) and the Calorimeter, will be ITER-like too, while the vacuum vessel will be without large flanges (differently from ITER) to reduce cost and weight. Only small flanges are foreseen on the vessel for pumping, diagnostics and BLC supplies (cooling water for all BLCs, $\mathrm{D}_{2}$ gas for neutralizer and electric bias for RID), while the BLC maintenance is planned to be from the large circular flanges of the beam sources, after removing the beam sources. For the vacuum pumping, it is foreseen to have a system based on turbomolecular pumps located on the side walls of the vessel and Non-Evaporable Getter (NEG) pumps [16] located on the upper and lower surfaces of the vessel.

The two beam sources have been conceptually designed to accelerate $40 \mathrm{~A}^{-}$of $\mathrm{D}^{-}$(20 A each) to an energy of $510 \mathrm{keV}$. This is made by means of a set of grids biased at different potentials (see Fig. 2 (c)): a Plasma Grid (PG) operating at $-510 \mathrm{kV}$, an Extraction Grid (EG) at $-500 \mathrm{kV}$, a Hyperlens Grid (HG) also at $-500 \mathrm{kV}$ (attached to the downstream side of the EG), a first Acceleration Grid (AG1) at $-333 \mathrm{kV}$, a second Acceleration Grid at $-166 \mathrm{kV}$ and a Grounded Grid (GG) at ground potential. In each of the two beam sources, all the grids are made with four copper segments to improve the beam aiming (each segment is in fact bent of about 1 degree with reference to the beam source axis, as shown in Fig. 3) and thermal deformation (all the segments have a fixed constraint in the central part of each grid, as shown in Fig. 2 (c)).

An extraction gap, about $6 \mathrm{~mm}$ long between PG and $\mathrm{EG}$, has the function to extract the $\mathrm{D}^{-}$ions from the plasma source and stop all the co-extracted electrons that are extracted together with the $\mathrm{D}^{-}$. In fact, these electrons would generate excessive heat loads on the following grids if they were not stopped by the EG while they still have a low energy. To do this, an electrostatic potential of about $10 \mathrm{kV}$ (varying in function of the density of the extracted $\mathrm{D}^{-}$to obtain good beam optics) is applied between the two grids and the EG is equipped with permanent magnets that deflect the co-extracted electrons onto the EG surface. Maximizing $\mathrm{D}^{-}$extraction while minimizing co-extracted electrons is one of the main critical issues for negative ion based NBIs. To do this, several methods are applied analogously to SPIDER, MITICA and ITER NBI $[17,18]$ :

- adopting an enhanced shape of the PG upstream sur- face (featuring large chamfers with a $40^{\circ}$ angle with reference to the beam axis);

- operating the PG at high temperature (about $150^{\circ} \mathrm{C}$ );

- covering the PG with a mono-layer of cesium, injected by dedicated cesium ovens mounted on the ion source;

- applying a high current flow vertically through the PG (this generates a filter field in front of the PG, as shown in Fig. 4 (b)).

After the extraction gap, there are three acceleration gaps with a thickness of about $100 \mathrm{~mm}$ and an applied potential of about $166 \mathrm{kV}$ each. The $\mathrm{D}^{-}$ions are accelerated passing through the apertures in each grid. Electrons, neutrals $\left(\mathrm{D}^{0}\right)$ and positive particles $\left(\mathrm{D}^{+}\right.$and $\left.\mathrm{D}_{2}^{+}\right)$are generated in the accelerator by stripping and charge-exchange reactions of the $\mathrm{D}^{-}$particles with the background gas, that is mainly $\mathrm{D}_{2}$. These particles, as well as the $\mathrm{D}^{-}$having a large divergence (called halo fraction of the beam) are finally impinging on the grids and on the plasma source, generating high heat fluxes on this surfaces and other backscattered particles. For this reason, all the grids and the plasma source components are equipped with high performance cooling systems able to avoid damages due to the extremely concentrated heat loads, and the density of the background gas in the accelerator is minimized by means of a high performance vacuum system mounted on vessel, composed by several NEG and turbo-molecular pumps. The pumping of the accelerator is enhanced by adding various holes on the flat part of the grid frames, so that the particles can flow to the pumped regions of the vessel.

Each grid segment is mounted on a stainless steel support frame, that in turn is connected to its external flange (also made of stainless steel). The external flanges are separated by insulating rings made of Fiber Reinforced Plastic (FRP) having an external diameter of about $2 \mathrm{~m}$ and designed to hold the potential difference of about $166 \mathrm{kV}$ between two adjacent flanges. In order to avoid discharges, the flanges are equipped with Electrostatic Screens (ES) and Triple Point Field Limiters (TPFL) on the vacuum side, and with Field Reducing Tubes (FRT) on the air side, as shown in Fig. 2 (b), analogously to the Japanese beam sources $[13,14]$.

The accelerator grid segments represent a critical component as they have a rather complex design, with very small cooling channels, apertures for the negative ions and grooves for the embedded magnets. They can be manufactured by means of copper electrodeposition on a milled base plate made of pure copper [19]. An alternative manufacturing process based on additive manufacturing is currently being investigated by means of a dedicated $R \& D$ program [20]. Another critical R\&D program is foreseen to develop and validate a manufacturing process for the FRP (fiber-reinforced polymer) rings of the accelerator. In fact, these rings are quite large (diameter of $2 \mathrm{~m}$ ) and must fulfill various important functions, i.e. to maintain the elec- 


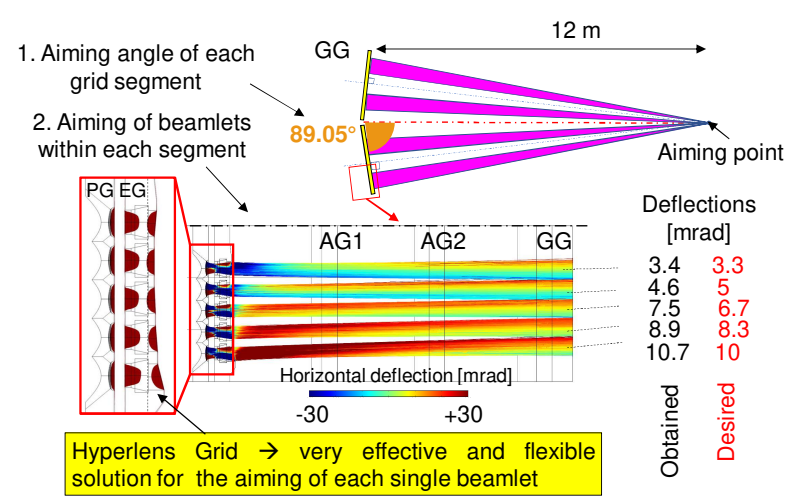

Fig. 3 Aiming strategy of the accelerator.

trical insulation between the acceleration stages and to support the beam source while being perfectly leak tight and having a vacuum compatible surface on the internal side.

\section{Aiming Strategy}

Each grid segment features two aperture groups having 95 apertures each arranged in a $19 \times 5$ matrix (partially shown in Fig. 2 (c)). As there are two beam sources having 4 grid segments each, the total number of apertures is 1520, through which 1520 beamlets (one per each aperture) are extracted and accelerated forming the $\mathrm{D}^{-}$beam. The geometry and layout of the apertures is derived from MITICA [18] to take advantage of the return of experience from ITER-related R\&D, but the apertures are about $18 \%$ more than in ITER NBI to decrease the requirement on the density of extracted $\mathrm{D}^{-}$current and thus increase the Availability index. To maximize the efficiency of the NBI, the beam optics must be optimized, i.e. the divergence of each beamlet must be minimized and a certain deflection must be applied to each beamlet so that each beamlet is aimed at the aiming point located in the tokamak, located about 200 $\mathrm{mm}$ downstream of the first wall and at a distance of $12 \mathrm{~m}$ from the GG.

The strategy adopted for the beam aiming is based on the inclination of the grid segments, as hinted in the previous paragraph, and on the usage of a Hyperlens Grid (HG) attached to the downstream side of the EG, as shown in Fig. 3. The HG was found to be the best option to provide a suitable deviation to each beamlet so that all the beamlets aim at the prescribed aiming point. This solution was found to be very effective in steering the beamlets, because also the steering of the beamlets located in the central part of the beamlet groups can be controlled precisely. The results of the first simulations with this solution are promising, as the obtained deflections for each beamlet are very close to the desired ones, as can be seen comparing the desired and obtained results reported in Fig. 3.

Several important parameters, i.e. wall-plug efficiency of the NBI, $\mathrm{D}_{2}$ gas throughput required at the neutralizer, requirements of the pumping system, heat loads on the
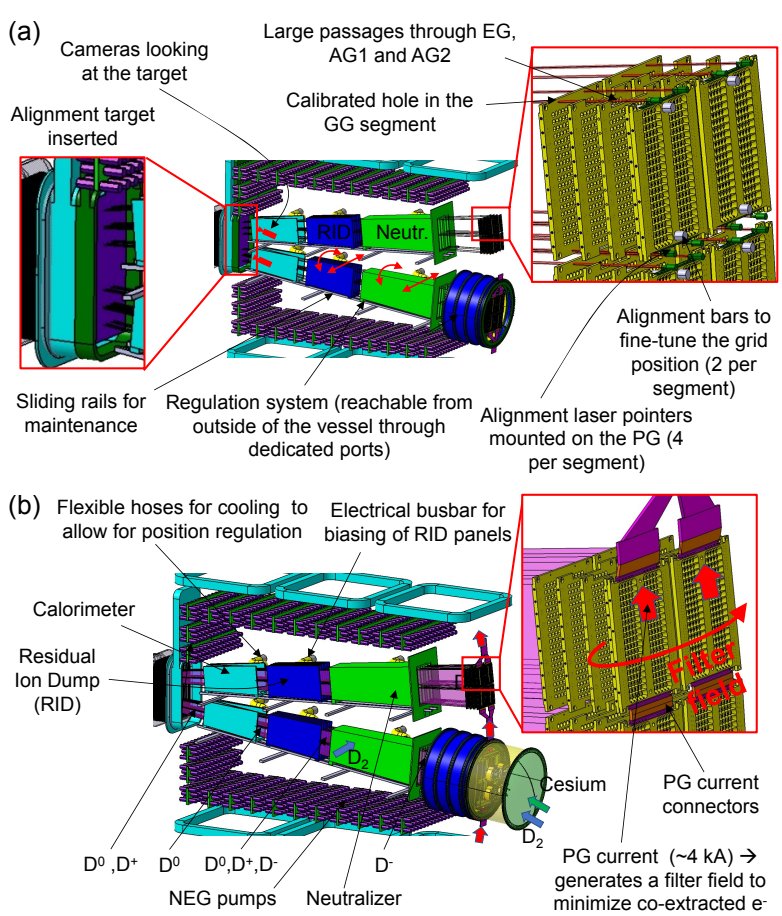

Fig. 4 Main operations stages of the beamline: (a) alignment of the components; (b) Beam injection in the plasma.

BLCs and duct, are strongly depending on the design of the BLCs and in particular on the distance between the beam and the panels. For this reason, comprehensive studies, including simulations of particle tracing, beam reactions and background particle flux in vacuum, are on-going to optimize the design of the BLCs.

\section{Alignment Strategy}

During the development of the NBI conceptual design, a large effort has been devoted to the optimization of the alignment procedure for the accelerator and beam line components, in order to minimize the time and effort needed for a suitable alignment before starting the beam operation and the manufacturing costs. This significantly impacts the RAMI indexes.

After considering several alignment strategies, it was chosen to equip the beamline with an alignment target located downstream of the calorimeter. During the alignment stage, a set of optical laser pointers (two per grid segment) are mounted on the PG, as shown in Fig. 4 (a). The optical laser passes through a calibrated hole in the GG, so that the laser downstream of the accelerator has the same direction as the ion beam. The footprints of the optical lasers can be observed by means of two dedicated visible cameras mounted on the vessel, so that the operator can check if they are in the correct position. If they are not in the correct position, the operator can move the whole set of segments (PG, EG, AG1, AG2 and GG) by means of two alignment bars that are passing through all the seg- 
ments using dedicated holes, as shown in Fig. 4 (a). When the correct position of the segments is obtained, the operator can block the position of each segment with respect to its support frame by means of a set of regulation screws mounted on the corresponding regulation pins (indicated in Fig. 2 (c)). The key point of this strategy is that the regulations screws of PG, EG AG1 and AG2 segments can be reached and tightened also when the whole accelerator is mounted by dedicated holes in the grids. This means that a fine-tuning of the beam aiming will be possible also after mounting the whole accelerator, allowing the possibility to manufacture the supporting frames, external flanges and insulating rings with relaxed mechanical tolerances, with a significant decrease of manufacturing cost, and to adopt a faster alignment procedure after each shut down.

Also the three BLCs need to be precisely aligned with reference to the beam, because their alignment with the beam affects several important operating parameters. This can be done by observing the footprints of the same optical lasers and moving the BLCs by means of movable supports reachable from outside the vessel (using dedicated portholes). In order to switch to beam-on operations, the optical lasers supports and the alignment bars will be removed from the PG, while the PG current connectors and the ion source assembly will be mounted, as shown in Fig. 4 (b). During the beam-on operations, the alignment target could be substituted by a carbon target able to absorb the beam for small periods (of the order of some seconds), observed by two thermo-cameras mounted instead of the previously mentioned visible cameras on the vessel lateral walls. In this way, it will be possible also to check the beam footprint during beam-on operations. This alignment strategy was found to strongly increase the Availability, Maintainability and Inspectability indexes, and is expected to significantly decrease the costs for the production and operation of the beamline.

\section{Conclusions}

A first complete conceptual design of the DTT NBI beamline has been developed considering $510 \mathrm{keV}$ beam energy and $10 \mathrm{MW}$ injected to the plasma, following a double beam source design approach. The main design guidelines have been the return of experience from existing experiments, the reduction of cost and the maximization of the RAMI indexes.

[1] DTT Divertor Tokamak Test facility Interim Design
Report, ENEA (ISBN 978-88-8286-378-4), April 2019 (“Green Book"), https://www.dtt-dms.enea.it/share/s/ avvglhVQT2aSkSgV9vuEtw

[2] R. Albanese et al., DTT: a divertor tokamak test facility for the study of the power exhaust issues in view of DEMO, Nucl. Fusion 57, 016010 (2017).

[3] R. Albanese et al., Design review for the Italian Divertor Tokamak Test facility, Fusion Eng. Des. 146, 194 (2019).

[4] G. Mazzitelli et al., Role of Italian DTT in the power exhaust implementation strategy, Fusion Eng. Des. 146, 932 (2019).

[5] G. Federici et al., Overview of the DEMO staged design approach in Europe, Nucl. Fusion 59, 066013 (2019).

[6] G. Granucci et al., The DTT device: System for heating, Fusion Eng. Des. 122, 349 (2017).

[7] P. Agostinetti et al., Conceptual design of a neutral beam heating \& current drive system for DTT, Fusion Eng. Des. 146, 441 (2019).

[8] J.F. Artaud et al., Metis: a fast integrated tokamak modelling tool for scenario design, Nucl. Fusion 58, 105001 (2018).

[9] R.B. White and M.S. Chance, Hamiltonian guiding center drift orbit calculation for plasmas of arbitrary cross section, Phys. Fluids 27, no. 10, 2455 (1984).

[10] TRANSP home page, https://transp.pppl.gov/index.html

[11] R.B. White, Chaos in trapped particle orbits, Phys. Rev. E 58, 1774 (1998).

[12] M. Gobbin et al., Numerical simulations of fast ion loss measurements induced by magnetic islands in the ASDEX Upgrade tokamak, Nucl. Fusion 49, 095021 (2009).

[13] A. Kojima et al., Progress in long-pulse production of powerful negative ion beams for JT-60SA and ITER, Nucl. Fusion 55, 063006 (2015).

[14] Y. Takeiri et al., High Performance of Neutral Beam Injectors for extension of LHD operational regime, Fusion Sci. Technol. 58, 482 (2010).

[15] B. Heinemann et al., Latest achievements of the negative ion beam test facility ELISE, Fusion Eng. Des. 136, 569 (2018).

[16] F. Siviero et al., Characterization of $\mathrm{ZAO}^{\circledR}$ sintered getter material for use in fusion applications, Fusion Eng. Des. 146, 1729 (2019).

[17] P. Agostinetti et al., Physics and engineering design of the Accelerator and Electron Dump for SPIDER, Nucl. Fusion 51, 063004 (2011).

[18] P. Agostinetti et al., Detailed design optimization of the MITICA negative ion accelerator in view of the ITER NBI, Nucl. Fusion 56, 016015 (2016).

[19] P. Agostinetti et al., Manufacturing and Testing of Grid Prototypes for the ITER Neutral Beam Injectors, IEEE Trans. Plasma Sci. 42, 628 (2014).

[20] M. Bonesso et al., Effect of particle size distribution on laser powder bed fusion manufacturability of copper, Proceedings of the 5th Metal Additive Manufacturing Conference 2020, pp. 173-182. 